\title{
Pathological characteristics of fifty patients with renal amyloidosis in Sri Lanka
}

\author{
D L P Dasanayaka ${ }^{1}$, S Wijetunge ${ }^{1}$, N V I Ratnatunga ${ }^{1}$
}

\begin{abstract}
Renal involvement is the most important cause of morbidity and mortality in systemic amyloidosis. This retrospective analysis was conducted to analyse the clinico-pathological characteristics of renal amyloidosis in a group of Sri Lankan patients undergoing renal biopsy. Renal amyloidosis was observed in 50/ $9712(0.5 \%)$ renal biopsies. The underlying cause for amyloidosis was not known in most. Of the known causes multiple myeloma was the commonest. Nephrotic range proteinuria was the most common clinical outcome and most had grade I to III chronic kidney disease at the time of diagnosis. Glomerulosclerosis was associated with the deterioration of renal function.
\end{abstract}

Ceylon Medical Journal 2016; 61: 189-190

http://doi.org/10.4038/cmj.v61i4.8388

\section{Introduction}

Renal amyloidosis is the most important cause of morbidity and mortality in systemic amyloidosis. In kidneys amyloid usually deposits in the glomeruli, however, arteries, tubules and interstitium may also get involved. Amyloid deposition causes damage resulting in chronic kidney disease (CKD) characterized by glomerulosclerosis, tubular atrophy and interstitial fibrosis and inflammation, which lead to end stage renal disease. The most common clinical presentation is proteinuria which is often within the nephrotic range.

Amyloidosis is characterised by accumulation of insoluble and indigestible hyaline material in the extracellular spaces due to misfolding of certain body proteins. Although there are more than 20 recognised chemical forms of amyloid, only three forms are commonly encountered, namely, amyloid light chain (AL) in primary amyloidosis associated plasma cell/B cell dyscrasias, serum amyloid A (AA) in reactive/secondary amyloidosis associated chronic inflammatory conditions such as rheumatoid arthritis, tuberculosis, bronchiectasis and transthyretin (TTR) associated with senile systemic amyloidosis and familial amyloid polyneuropathies. Although amyloid has varied chemical compositions they share similar physical and staining properties due to structural similarities of the molecule. Confirmation of tissue amyloid deposition is made histologically by congo red staining and visualisation under polarized light.

Although renal amyloidosis is not uncommon, there are no reports about the clinico-pathological characteristics of renal amyloidosis in Sri Lanka. Hence we conducted a study to determine the pathological features of renal amyloidosis in Sri Lankan patients undergoing renal biopsy and correlated them with the clinical charactories.

\section{Methods}

This is a retrospective analysis of patients with histological diagnosis of renal amyloidosis. We analysed data of patients referred to the Department of Pathology, Faculty of Medicine, University of Peradeniya from 1993 to 2014. Amyloidosis was diagnosed using congo red stain visualised underpolarized light. Clinical and histological details were retrieved from the records. Glomerular filtration rate (GFR) was calculated using the modified diet formula. CKD was graded using clinical practice guidelines from stage I (GFR $>90 \mathrm{ml} / \mathrm{min}$ ) to stage $\mathrm{V}$ (GFR $<15 \mathrm{ml} / \mathrm{min}$ ). The degree of amyloid deposition in the kidney was graded as mild, moderate and severe according to the number of glomeruli involved, amount of amyloid in the glomeruli and presence of the same in the arterial walls and tubulo- interstitium. The degree of amyloid deposition and the percentage of glomerulosclerosis were correlated with GFR using Kendall's tau-b test and Spearman correlation respectively.

\section{Results}

Of 9712 renal biopsies assessed, $50(0.52 \%)$ had histological diagnosis of renal amyloidosis. The mean age of the patients was $56.7 \pm 9.4$ years (range 33 to 80 years) and $25(50 \%)$ were males. Proteinuria was the presenting abnormality in 48 and 27 (54\%) of them had

${ }^{1}$ Department of Pathology, Faculty of Medicine, University of Peradeniya, Sri Lanka.

Correspondence: SW, e-mail: <suwijetunge@gmail.com>. Received 18 January 2016 and revised version accepted 25 August 2016. and reproduction in any medium, provided the original author and source are credited. 
nephrotic range proteinuria. Eighteen $(36 \%)$ had stage I or II CKD, $15(30 \%)$ stage III and $5(10 \%)$ stage IV or $\mathrm{V}$ disease; GFR was not known in the rest $(24 \%)$. Six (12\%) had confirmed multiple myeloma and $15(30 \%)$ were under investigation for the condition. One (2\%) had history of pulmonary tuberculosis and the cause was unknown at the time of diagnosis in the rest (56\%). Histologically, 49 had glomerular amyloid, of them 33 had arteriolar wall deposits and 4 had tubular and interstitial deposits as well. One had interstitial amyloid deposits only. Degree of amyloid deposition was mild in 5 , moderate in 11 and severe in the rest. Mean percentage of glomerulosclerosis was $17.9 \%( \pm 23.3)$. Tubular atrophy and chronic interstitial inflam-mation and fibrosis of varying degree were present in 46 . The rest did not show tubulo-interstitial changes. One had evidence of cast nephropathy as well. There was statistically significant correlation between the percentage of glomerulosclerosis and GFR $(\mathrm{r}=-0.44 ; p=0.006)$. The correlation between the severity of amyloid deposition and GFR $(r=-0.03)$ was not statistically significant $(p=0.843)$.

\section{Discussion}

In the present study we found that the severity of renal amyloid deposition does not correlate with the severity of renal impairment, which has been shown before $[1,2]$. However, the degree of renal impairment correlated with severity of glomerulosclerosis which is a secondary effect of amyloidosis induced renal damage. These observations challenge the long held belief that renal damage in amyloidosis is caused by tissue disruption due to pressure effects of amyloid. Several studies have demonstrated that amyloid precursor proteins themselves have tissue toxicity and therefore, may play a role in the pathogenesis of tissue damage $[3,4]$.

In renal amyloidosis, as observed in the present study too, amyloid is predominantly deposited in the glomeruli with or without involvement of the arteries and tubulointerstitium, while tubulo-interstitial only disease is rare [5]. Nephrotic or subnephrotic proteinuria is the usual clinical presentation in glomerular disease and in the rare tubulo-interstitial only disease gradually declining GFR is the usual clinical manifestation. However, in the present study, the one patient with interstitial only disease had subnephrotic range proteinuria.

Twenty one (42\%) with renal amyloidosis had either confirmed or suspected multiple myeloma and, apart from the one with tuberculosis, others did not have an apparent cause at the time of diagnosis. Worldwide the commonest cause of renal amyloidosis is multiple myeloma/plasma cell dyscrasia followed by chronic inflammatory diseases [5]. Other causes of systemic amyloidosis such as haemodialysis associated amyloidosis does not affect the kidneys, senile amyloidosis predominantly affect the heart and the familial amyloidosis is rare in Asia $[5,6]$. In the present study majority, of those with a known aetiology, had multiple myeloma associated and it is likely that in many of those without an apparent aetiology too the underlying disease could be multiple myeloma/ plasma cell dyscrasia. However, follow up details of these patients were not available. The main limitation in the present study was that the clinical details obtained were limited to those provided in the request form.

In conclusion, glomerulosclerosis determined the deterioration of renal function rather than the severity of amyloid deposition in this cohort of patients from Sri Lanka.

\section{Acknowledgements}

Authors thank doctors TDJ Abeysekara, AWM Wazil, N Nanayakkara, M Selavarajah and Dr R. Dissanayaka for sending the renal biopsies and GHDS Chandraprabha and $\mathrm{N}$ Herath for the technical support.

\section{Conflicts of interest}

There are no conflicts of interest

\section{References}

1. Lobato L, Beirao I, Guimaraes SM, et al. Familial amyloid polyneuropathy type I (Portuguese): Distribution and characterization of renal amyloid deposits. Am J Kidney Dis 1998; 31: 940-6.

2. Snanoudj R, Durrbach A, Gauthier E, et al. Changes in renal function in patients with familial amyloid polyneuropathy treated with orthotopic liver transplantation. Nephrol Dial Transplant 2004; 19: 1779-85.

3. Brenner DA, Jain M, Pimentel DR, et al. Human amyloidogenic light chains directly impair cardiomyocyte function through an increase in cellular oxidant stress. Circ Res 2004; 94: 1008-10.

4. Sousa MM, Cardoso I, Fernandes R, Guimaraes A, Saraiva MJ. Deposition of transthyretin in early stages of familial amyloidotic polyneuropathy: Evidence for toxicity of nonfibrillar aggregates. Am J Pathol 200; 159: 1993-2000.

5. Dember L. M. Amyloidosis-Associated Kidney Disease. J Am Soc Nephrol 2006; 17: 3458-71.

6. Galappatthy P, Wijeweera I, Wijesekara JC, Kodikara DR, Fonseka HC. Familial amyloid polyneuropathy in a Sri Lankan family. Ceylon Med J 1997; 42: 33-5. 